\title{
Synthesis of Formylchromone Derivatives; Inactivators of Protein Tyrosine Phosphatase 1B
}

\author{
Suja Shrestha, Su Yeon Hwang, Keun-Hyeung Lee, and Hyeongjin Cho* \\ Department of Chemistry and Institute of Molecular Cell Biology, Inha University, Incheon 402-751, Korea \\ *E-mail: hcho@inha.ac.kr \\ Received December 30, 2004
}

Key Words : Formylchromone, Protein tyrosine phosphatase, PTP1B, Inactivator

Protein tyrosine phosphatases (PTPases) represent a family of enzymes that hydrolyze phosphate moiety from phosphotyrosine residues of cellular proteins ${ }^{1}$ and they constitute essential components for the dynamic and precise control of the phosphorylation level of signaling proteins., ${ }^{2,3}$ Because of the importance of tyrosine phosphorylation in signal transduction, PTPases have emerged as potential targets for the treatment of a diverse of human diseases. ${ }^{4-6}$ For example, inhibition of protein tyrosine phosphatase $1 \mathrm{~B}$ (PTP1B) might be a strategy to compensate the defective insulin sensitivity commonly observed in the patients of type II diabetes. ${ }^{7,8}$ In an effort to find potent and specific inhibitors of PTPases, we found that 3-formylchromone (4oxo-4H-[1]-benzopyran-3-carboxaldehyde, 3-formyl-4benzopyrone) derivatives 1 inactivate PTPases. Some of them were potent and selective against PTP1B and this result was reported recently. ${ }^{9,10}$ Hereby, we report the synthesis of the formylchromone derivatives.

One of the synthetic strategy involves the preparation of appropriately substituted phenol derivatives $\mathbf{3}$ followed by Friedel-Crafts acylation to obtain 2-acetylphenol derivatives 7 (top line of Scheme 1). Alternatively, 7 could be obtained by acetylation prior to the introduction of the substituent $\mathrm{R}$ (second line of Scheme 1). The core structure was then formed by Vilsmeier-Haack reaction in which 2-acetylphenol moiety in 7 was converted to 3-formylchromone structure in the presence of $\mathrm{POCl}_{3}$ and DMF (Scheme 1). ${ }^{11-13}$ Other routes have been precedented for the construction of 3-formylchromone, but the most convenient was the method via Vilsmeier-Haack reaction. ${ }^{14}$

Synthesis of the PTPase inactivators are summarized in Schemes 2-7. For the synthesis of TP14, the anthracene

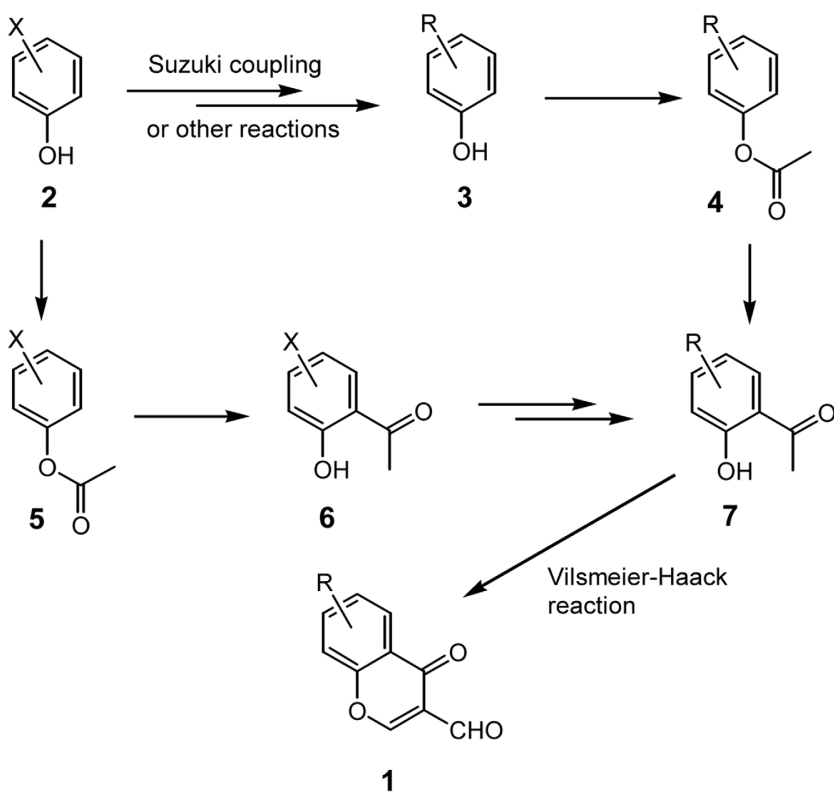

Scheme 1. Synthetic strategy.

structure was constructed by reaction of anthrone with anisyl magnesium bromide followed by concomitant aromatization of the ring. ${ }^{15}$ Acetylation at the ortho-position of the hydroxy group was accomplished by converting the phenolic compound 14-3 into acetate 14-4 followed by heating with $\mathrm{AlCl}_{3}$. Friedel-Crafts acylation of phenol derivatives under usual reaction condition $\left(\mathrm{Ac}_{2} \mathrm{O}\right.$ or $\left.\mathrm{AcCl}, \mathrm{AlCl}_{3}\right)$ complicated the reaction by acetylation at unwanted position. Vilsmeier-Haack reaction of the 2-acetylphenol derivative 14-6 completed the synthesis.

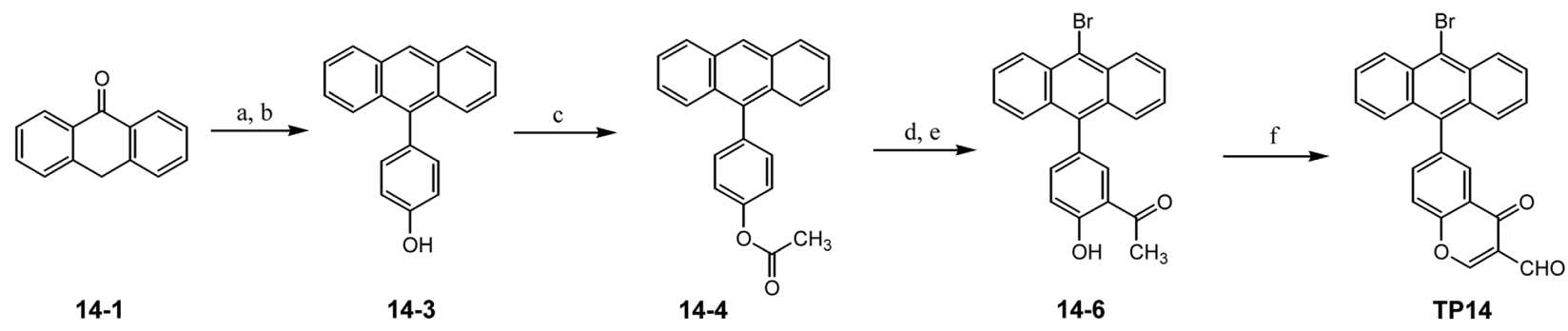

Scheme 2. Reagents and conditions: (a) $\mathrm{CH}_{3} \mathrm{OC}_{6} \mathrm{H}_{5} \mathrm{MgBr}$, benzene, $10{ }^{\circ} \mathrm{C} \rightarrow 70{ }^{\circ} \mathrm{C}, 69 \%$; (b) $\mathrm{BBr}_{3}, \mathrm{CH}_{2} \mathrm{Cl}_{2}, 96 \%$; (c) $\left(\mathrm{CH}_{3} \mathrm{CO}\right)_{2} \mathrm{O}$, pyridine, $\mathrm{CH}_{2} \mathrm{Cl}_{2}, 75 \%$; (d) $\mathrm{AlCl}_{3}, 1,2$-Dichlorobenzene, $100{ }^{\circ} \mathrm{C}$; $33 \%$; (e) $\mathrm{Br}_{2}, \mathrm{Et}_{2} \mathrm{O}, 98 \%$; (f) $\mathrm{POCl}_{3}, \mathrm{DMF},-20^{\circ} \mathrm{C} \rightarrow 50{ }^{\circ} \mathrm{C}, 3 \mathrm{~h}, 50 \%$. 


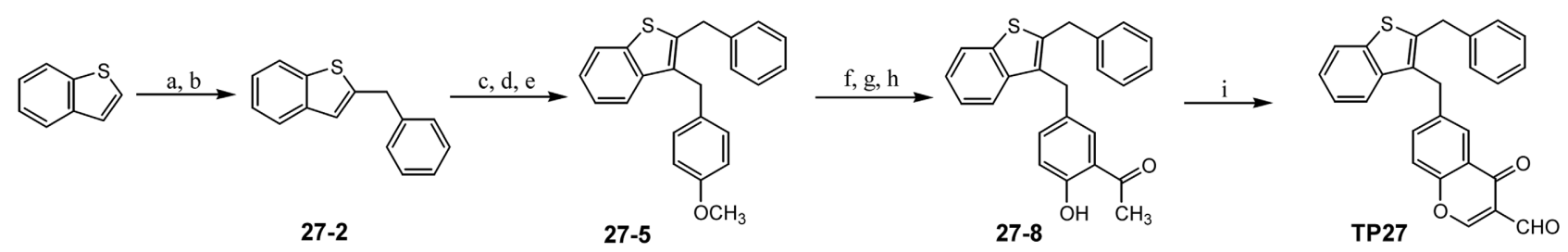

Scheme 3. Reagents and conditions: (a) i) n-BuLi, THF, $-78{ }^{\circ} \mathrm{C}$; ii) benzaldehyde, $-78{ }^{\circ} \mathrm{C} \rightarrow$ r.t., $91 \%$; (b) $\mathrm{NaBH}_{4}, \mathrm{Et}_{2} \mathrm{O}$, then 18 equiv. $\mathrm{CF}_{3} \mathrm{COOH}, 98 \%$; (c) $p$-Anisoylchloride, $\mathrm{CS}_{2}, 89 \%$; (d) $\mathrm{LiAlH}_{4}, \mathrm{Et}_{2} \mathrm{O}, 99 \%$; (e) $\mathrm{NaBH}_{4}, \mathrm{Et}_{2} \mathrm{O}$, then 3.7 equiv. $\mathrm{CF}_{3} \mathrm{COOH}_{2}, 99 \%$; (f) $\mathrm{BBr}_{3}$, $\mathrm{CH}_{2} \mathrm{Cl}_{2}, 99 \%$; (g) $\left(\mathrm{CH}_{3} \mathrm{CO}\right)_{2} \mathrm{O}$, pyridine, $\mathrm{CH}_{2} \mathrm{Cl}_{2}, 81 \%$; (h) $\mathrm{AlCl}_{3}, 1,2$-Dichlorobenzene, $95{ }^{\circ} \mathrm{C}$; $33 \%$; (i) $\mathrm{POCl}, \mathrm{DMF}_{3}-20^{\circ} \mathrm{C} \rightarrow 50{ }^{\circ} \mathrm{C}, 2 \mathrm{~h}$, $67 \%$.

Skeletal construction for TP27 was accomplished by two consecutive acylation/reduction of benzothiophene (Scheme 3). Regiospecific benzoylation at C-2 of benzothiophene followed by reduction of the carbonyl group afforded 27-2, which in turn acylated under Friedel-Crafts condition and then reduced to obtain 27-5. ${ }^{16-18}$ The rest of the steps for the synthesis of TP27 are the same as those for TP14; removal of the methyl protecting group, O-acetylation, rearrangement under Friedel-Crafts condition and Vilsmeier-Haack reaction as the last step.

Synthesis of TP30 and TP32 involves bromination and Suzuki coupling steps to introduce phenyl substituents (Scheme 4 and 5). ${ }^{19,20}$ Pinacol ester of phenylboronic ester was an effective reagent for the coupling reaction resulting in better yields compared to phenylboronic acid. Controlled bromination of 30-2 afforded monobrominated product 30-4 or dibrominated compound 32-1 selectively and they were subjected to Suzuki reaction. For the synthesis of TP32,

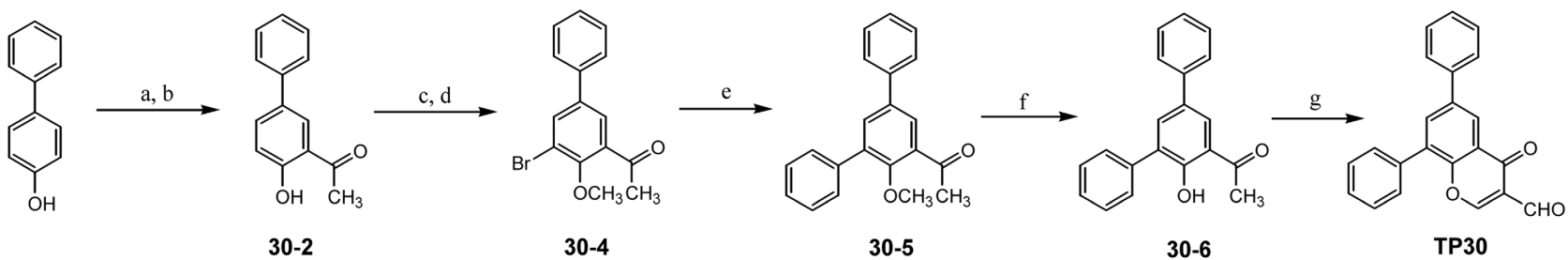

Scheme 4. Reagents and conditions: (a) $\left(\mathrm{CH}_{3} \mathrm{CO}\right)_{2} \mathrm{O}$, pyridine, $\mathrm{CH}_{2} \mathrm{Cl}_{2}, 86 \%$; (b) $\mathrm{AlCl}_{3}$, neat, $180{ }^{\circ} \mathrm{C}, 30 \mathrm{~min} ; 40 \%$; (c) $\mathrm{Br}_{2}, \mathrm{CH}_{2} \mathrm{Cl}_{2}, 77 \%$; (d) $\mathrm{CH}_{3} \mathrm{I}, \mathrm{K}_{2} \mathrm{CO}_{3}$, acetone, $50{ }^{\circ} \mathrm{C}, 90 \%$; (e) 4,4,5,5-tetramethyl-1,3,2-dioxaborolan-2-ylbenzene, $\mathrm{K}_{3} \mathrm{PO}_{4}, \mathrm{Pd}_{\left(\mathrm{PPh}_{3}\right)}$, $\mathrm{DMF}^{\circ}, 80{ }^{\circ} \mathrm{C}$, $86 \%$; (f) $\mathrm{BBr}_{3}, \mathrm{CH}_{2} \mathrm{Cl}_{2}, 90 \%$; (g) $\mathrm{POCl}_{3}, \mathrm{DMF},-20^{\circ} \mathrm{C} \rightarrow 50{ }^{\circ} \mathrm{C}, 4 \mathrm{~h}, 57 \%$.

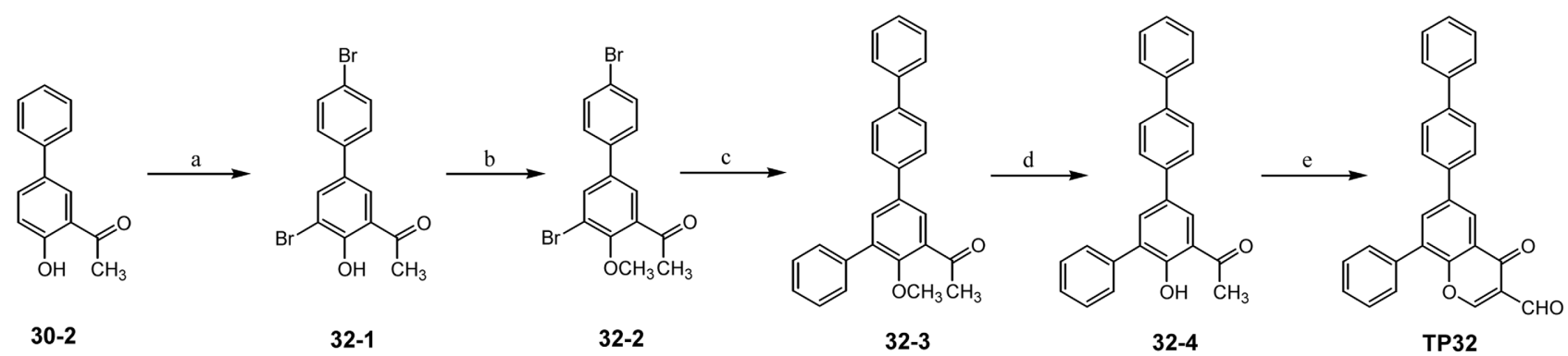

Scheme 5. Reagents and conditions: (a) $\mathrm{Br}_{2}, \mathrm{CH}_{2} \mathrm{Cl}_{2}, 60 \%$; (b) $\mathrm{CH}_{3} \mathrm{I}, \mathrm{K}_{2} \mathrm{CO}_{3}$, acetone, $40{ }^{\circ} \mathrm{C}, 73 \%$; (c) 4,4,5,5-tetramethyl-1,3,2dioxaborolan-2-ylbenzene, $\mathrm{K}_{3} \mathrm{PO}_{4}, \mathrm{Pd}\left(\mathrm{PPh}_{3}\right)_{4}$, DMF, $80{ }^{\circ} \mathrm{C}, 70 \%$; (d) $\mathrm{BBr}_{3}, \mathrm{CH}_{2} \mathrm{Cl}_{2}, 84 \%$; (e) $\mathrm{POCl}_{3}, \mathrm{DMF}^{\circ},-20{ }^{\circ} \mathrm{C} \rightarrow 50{ }^{\circ} \mathrm{C}, 4 \mathrm{~h}, 77 \%$.

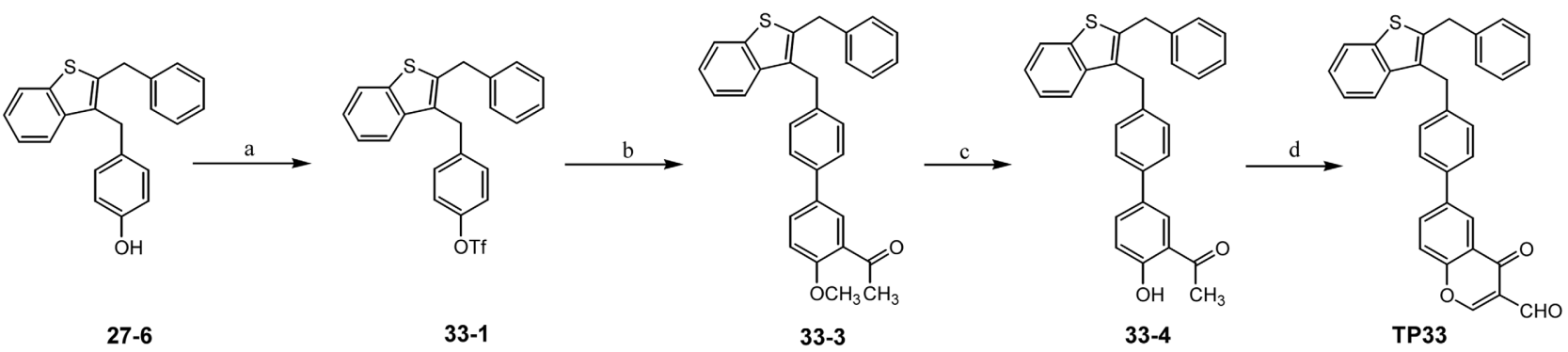

Scheme 6. Reagents and conditions: (a) $\left(\mathrm{CF}_{3} \mathrm{SO}_{2}\right)_{2} \mathrm{O}, 2$ 2,6-lutidine, $\mathrm{CH}_{2} \mathrm{Cl}_{2}, 75 \%$; (b) 2-methoxy-5-(4,4,5,5-tetramethyl-1,3,2-dioxaborolan-2-yl)acetophenone, $\mathrm{K}_{3} \mathrm{PO}_{4}, \mathrm{PdCl}_{2}$ (dppf), DMF, $80^{\circ} \mathrm{C}, 90 \%$; (c) $\mathrm{BBr}_{3}, \mathrm{CH}_{2} \mathrm{Cl}_{2}, 93 \%$; (d) $\mathrm{POCl}_{3}$, DMF, r.t. $\rightarrow 50{ }^{\circ} \mathrm{C}, 2 \mathrm{~h}, 41 \%$. 


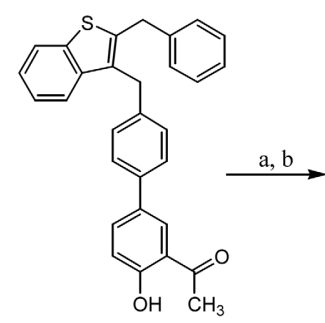

33-4

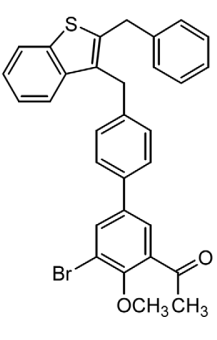

34-2

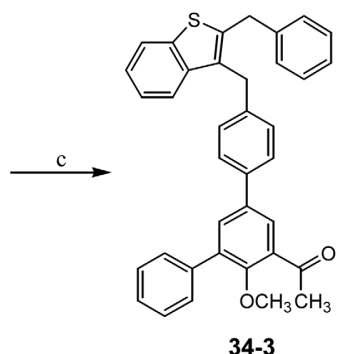

34-3
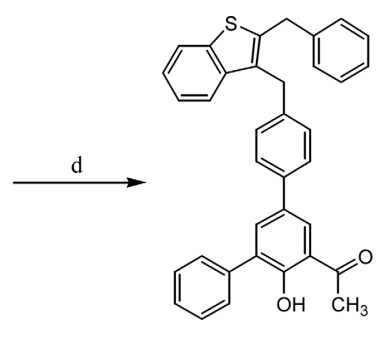

34-4

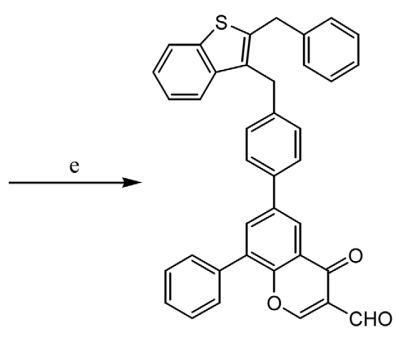

TP34

Scheme 7. Reagents and conditions: (a) $\mathrm{Br}_{2}$, acetate buffer, $\mathrm{pH} 3.7,93 \%$; (b) $\mathrm{CH}_{3} \mathrm{I}, \mathrm{K}_{2} \mathrm{CO}_{3}$, acetone, $40{ }^{\circ} \mathrm{C}$, 94\%; (c) 4,4,5,5-tetramethyl1,3,2-dioxaborolan-2-ylbenzene, $\mathrm{K}_{3} \mathrm{PO}_{4}, \mathrm{Pd}\left(\mathrm{PPh}_{3}\right)_{4}, \mathrm{DMF}, 80^{\circ} \mathrm{C}, 84 \%$; (d) $\mathrm{BBr}_{3}, \mathrm{CH}_{2} \mathrm{Cl}_{2}, 87 \%$; (e) $\mathrm{POCl} 3$, DMF, r.t. $\rightarrow 50{ }^{\circ} \mathrm{C}, 4$ h, $71 \%$.

double coupling reaction was performed to obtain 32-3.

Synthesis of TP33 (Scheme 6) was branched from the intermediate 27-6 in the synthesis of TP27. Triflate ester 33-1 was treated with boronate ester of protected 2-acetylphenol to obtain the coupling product 33-3. After demethylation, 33-4 was subjected to Vilsmeier-Haack reaction. The boronate ester was prepared by reaction of 2-methoxy-5bromoacetophenone with bis(pinacolato)diboron. ${ }^{19}$ Compound TP34 is an extended version of TP33 with an additional phenyl substituent and, therefore, its synthesis involves another Suzuki coupling reaction step (Scheme 7).

The compounds synthesized in this study inactivated 50\% of the $p$-nitrophenol phosphatase activity of PTP1B at low micromolar concentrations $\left(\mathrm{IC}_{50}\right.$ values: TP14, $2.5 \mu \mathrm{M}$; TP27, $3.2 \mu \mathrm{M}$; TP30, $3.3 \mu \mathrm{M}$; TP32, $2.0 \mu \mathrm{M}$; TP33, 1.1 $\mu \mathrm{M}$; TP34, $1.0 \mu \mathrm{M}$ respectively). Biochemical data of those together with dozens of less potent formylchromone derivatives were reported in separate publications. ${ }^{9,10}$

Formylchromones have been of synthetic and biological interest because of their synthetic utility based on the three electrophilic carbon atoms and the biological activities associated with the formylchromone derivatives or structurally modified compounds. ${ }^{21-23}$ In this study, we have described the synthesis of a variety of 3-formylchromone derivatives which behaved as potent and selective inactivators of PTP1B. This preparation extends the synthetic utility of 3-formylchromones and provides easy access to the compounds for medicinal or biological application.

Supplementary Materials. Detailed experimental procedures and the spectral data of the compounds are available from the author upon request.

Acknowledgements. This work was supported by the grant from Inha University (2005). S. Shrestha is a recipient of BK21 fellowship.

\section{References}

1. Zhang, Z.-Y. Annu. Rev. Pharmacol. Toxicol. 2002, 42, 209.

2. Cohen, P. Trends Biochem. Sci. 2000, 25, 596.

3. Neel, B. G.; Tonks, N. K. Curr. Opin. Cell Biol. 1997, 9, 193.

4. Van Huijsduijnen, R. H.; Bombrun, A.; Swinnen, D. Drug Discov. Today 2002, 7, 1013.

5. Zhang, Z.-Y. Curr. Opin. Chem. Biol. 2001, 5, 416.

6. Li, L.; Dixon, J. E. Semin. Immunol. 2000, 12, 75.

7. Elchebly, M.; Payette, P.; Michaliszyn, E.; Cromlish, W.; Collins, S.; Loy, A. L.; Normandin, D.; Cheng, A.; Himms-Hagen, J.; Chan, C. C.; Ramachandran, C.; Gresser, M. J.; Tremblay, M. L.; Kennedy, B. P. Science 1999, 283, 1544.

8. Zinker, B. A.; Rondinone, C. M.; Trevillyan, J. M.; Gum, R. J.; Clampit, J. E.; Waring, J. F.; Xie, N.; Wilcox, D.; Jacobson, P.; Frost, L.; Kroeger, P. E.; Reilly, R. M.; Koterski, S.; Opgenorth, T. J.; Ulrich, R. G.; Crosby, S.; Butler, M.; Murray, S. F.; McKay, R. A.; Bhanot, S.; Monia, B. P.; Jirousek, M. R. Proc. Natl. Acad. Sci. U. S. A. 2002, 99, 11357.

9. Shim, Y. S.; Kim, K. C.; Lee, K. A.; Shrestha, S.; Lee, K.-H.; Kim, C. K.; Cho, H. Bioorg. Med. Chem. 2005, 13, 1325.

10. Shim, Y. A.; Kim, K. C.; Chi, D. Y.; Lee, K.-H.; Cho, H. Bioorg. Med. Chem. Lett. 2003, 13, 2561

11. Nohara, A.; Umetani, T.; Sanno, Y. Tetrahedron 1974, 30, 3553.

12. Nohara, A.; Umetani, T.; Sanno, Y. Tetrahedron Lett. 1973, 22, 1995.

13. Reynolds, G. A.; Van Allan, J. A. J. Heterocyclic Chem. 1969, 6, 375.

14. Sabitha, G. Aldrichim. Acta 1996, 29, 15.

15. Schiessler, R. W.; Rytina, A. W.; Whitmore, F. C. J. Am. Chem. Soc. 1948, 70, 529 .

16. Gribble, G. W.; Leese, R. M. Synthesis 1977, 172.

17. Nutaitis, C. F.; Patragnoni, R.; Goodkin, G.; Neighbour, B.; Obaza-Nutaitis, J. Org. Prep. Proced. Int. 1991, 23, 403.

18. Wrobel, J.; Sredy, J.; Moxham, C.; Dietrich, A.; Li, Z.; Sawicki, D. R.; Seestaller, L.; Wu, L.; Katz, A.; Sullivan, D.; Tio, C.; Zhang, Z.-Y. J. Med. Chem. 1999, 42, 3199.

19. Suzuki, A. J. Organomet. Chem. 1999, 576, 147.

20. Miyaura, N.; Suzuki, A. Chem. Rev. 1995, 95, 2457.

21. Nakano, K.; Nakayachi, T.; Yasumoto, E.; Morshed, S. R. M. D.; Hashimoto, K.; Kikuchi, H.; Nishikawa, H.; Sugiyama, K.; Amano, O.; Kawase, M.; Sakagami, H. Anticancer Res. 2004, 24, 711.

22. Foltnova, P.; Lacova, M.; Loos, D. Il Farmaco 2000, 55, 21.

23. Hafez, M. E.-S.; Pavlina, F.; Margita, L.; Jarmila, C.; Henrieta, S. Il Farmaco 1998, 53, 224. 